\title{
OPTIMIZING BIAS POINT OF HIGH EFFICIENCY CLASS-B GAN POWER AMPLIFIER FOR THE BEST EFFICIENCY
}

\author{
Ondrej Fiser \\ Doctoral Degree Programme (1), FEEC BUT \\ E-mail: xfiser13@vutbr.cz \\ Supervised by: Tomas Gotthans \\ E-mail: gotthans@vutbr.cz
}

\begin{abstract}
High performance amplifiers are always a demanding component in the world of wireless communication. The amplifier is the heart that drives each radio system. We have designed and developed a high performance one-stage class-B GaN power amplifier for drone applications in the S-band (at 1,6 GHz) with maximum output power $6 \mathrm{~W}$. This paper compare fixed settings of the bias point option and optimized bias point for the best efficiency within the entire output power range. Applying the proposed method, that is particularly advantageous for low power performance to improve efficiency by more than $15 \%$.
\end{abstract}

Keywords: GaN, S-band, RF power amplifier, Class-B, PAE, Bias circuit, Matlab

\section{INTRODUCTION}

Efficiency and linearity of the microwave power amplifier (PA) are critical elements for modern wireless communication systems. An optimal PA is designed on the following parameters: Gain, PAE (Power-Added Efficiency), RF power, ACPR (Adjacent Channel Power Ratio), linearity, stability and etc. To minimize distortion, they are typically operated in Class-A or Class-AB mode. Unfortunately, the efficiency of these classes is very low and therefore not suitable for input signals with a high peak to average power ratio (PAPR). For example in the case of OFDM (Orthogonal Frequency Division Multiplexing) we can have $12 \mathrm{~dB}$ PAPR and in order to amplify the signal without distortions, back off must be applied [3].. This problem can solve the use of, for example, Doherty's topology [1] that combines the amplifier class- $\mathrm{AB}$ and $\mathrm{C}$, where majority of the signals are amplified in the $\mathrm{AB}$ class and the peaks are amplified in class-C. On the other hand, the Doherty architecture is not broadband. An usual amplifier works with a fixed supply voltage (on the transistor drain) and a fixed bias voltage at the gate of transistor. With the advent of fast switching elements, drain voltage can be changed dynamically, depending, for example, on the input power (known as envelop tracking [4], [6]), temperature or other parameters, thereby further enhancing the efficiency of the amplifier.

In this paper, the aim is focused on optimization of bias point of PA in class-B for the best efficiency within the entire output power range. We designed one-stage PA (which was designed and developed before) for airborne application with RF Power GaN HEMT transistor CGH40006P from CREE Corporation. The basic idea of this paper is that if the airplane is near a station or satellite with which it communicates (and has good conditions for communication), it does not need to transmit at maximum power (from the point of view of the energy balance). For example in the close proximity, usually the output power is derated by decreasing the input power. But, such approach will use the PA in inefficient region causing high raise of junction temperature. Therefore we propose instead of decreasing the input power, to change the supply voltages.

The paper is organized as follows: section II contains design of PA and Section III a brief description of the method for optimizing best of bias point. The measurements, laboratory setup and obtained 
results are described in Section IV. Section V closes this paper.

\section{PA DESIGN}

In PA design, Microwave office (AWR-R14) software from National Instruments is used. For the design, a nonlinear model of CGH40006P from CREE Corporation was used with common source configuration. We designed a broadband c1ass-B amplifier in the carrier frequency 1,6 GHz.

Cree (now known as Wolfspeed) GaN (Gallium Nitride) HEMTs (High Electron Mobility Transistors) offers greater power density and wider bandwidths compared to $\mathrm{Si}$ and GaAs (Gallium Arsenide) transistors. GaN has superior properties compared to silicon or gallium arsenide, including higher breakdown voltage, higher saturated electron drift velocity, and higher thermal conductivity.

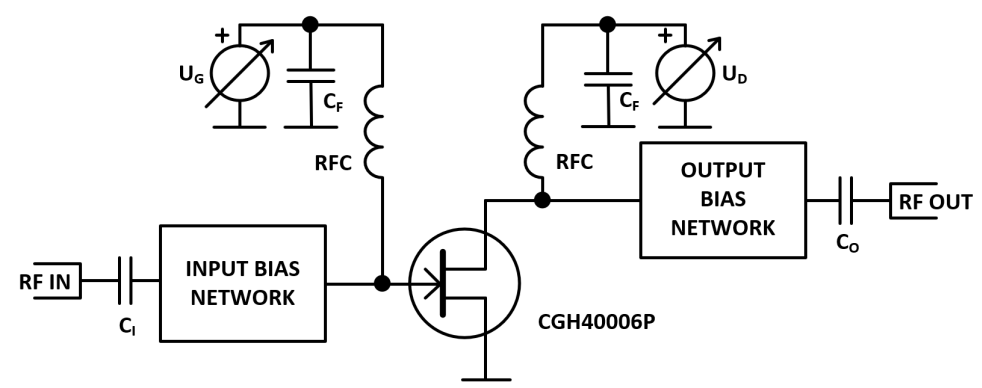

Figure 1: Schematic of c1ass-B amplifier

\subsection{QUIESCENT POINT SELECTION}

For an amplifier in c1ass-B (Fig. 1), it is typical to select the quiescent point (Q-point) near pinch-off voltage [5]. Q-point is selected just below the pinch-off at $\mathrm{V}_{G S}=-2.85 \mathrm{~V}\left(\mathrm{I}_{D S}=81.4 \mathrm{~mA}\right)$ and the drain voltage is $28 \mathrm{~V}$.

Importantly, during the power up and initial adjustment testing, the Q-point is set according to the drain current, because the real $\mathrm{U}_{G S}$ can be slightly different from the simulation, because the simulation rely on a transistor model and not on a load-pull measurement.

\subsection{BROADBAND MATCHING NETWORK DESIGN}

After Q-point selection for class-B operation, another important task in PA design is to select proper bias network in order to avoid any oscillation, interference and too match input/output. A big advantage of the used transistor is total stability over the entire frequency range $1-6 \mathrm{GHz}$. The output impedance of the transistor was determined Load-Pull simulation of the amplifier (Fig. 2a), which consisted in sweeping the reflection factor on the output and monitoring the change of important parameters - output level, efficiency, phase distortion and linearity (Fig. 2b). The input impedance of the transistor was determined based on parameter S11.

Based on the found input and output impedance of the transistor, an input and output matching network for $50 \mathrm{Ohms}$. With respect to the power and high operating frequency of the amplifier, the matching circuits are formed by microstrip lines with a blocking capacitor for the DC component.

The final phase of the amplifier design is the design of its bias circuitry for gate and drain of the transistor. Bias circuit supplies DC voltage and prevents the penetration of the RF signal to the DC amplifier section. The bias circuit for the gate can be designed easily by using high-conduction line, 


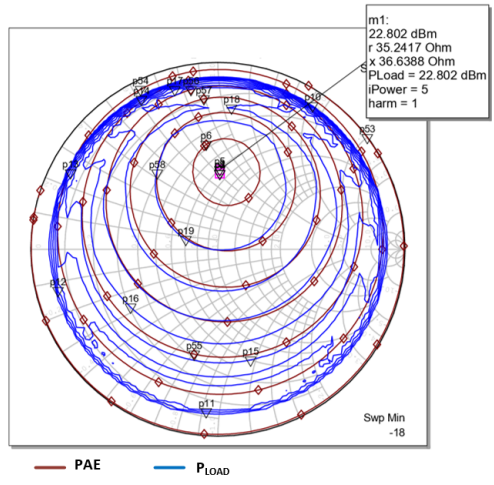

(a)

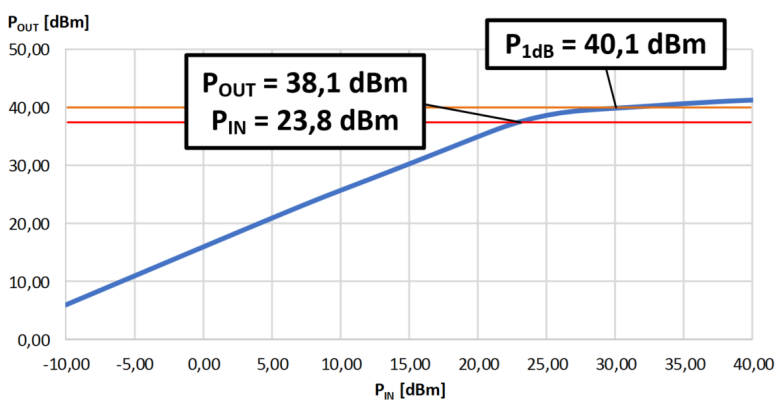

(b)

Figure 2: a) Simulated load-pull analyze - marker indicates maximum PAE and output power. b) Simulated AM-AM linearity analyze.

typically $\geq 100$ Ohms. In contrast, the bias circuit for the drain of the transistor must handle the DC current in order of amperes. In this design of amplifier, bias circuits are implemented by two RF chokes, which perceive the RF signal as high impedance and do not penetrate the DC part of the amplifier.

Hence, the final schematic of class-B PA design is shown in Fig. 1. After design, PA was fabricated on FR4 substrate, thickness of the substrate was $1 \mathrm{~mm}$ and dielectric constant $\left(\varepsilon_{r}\right)$ was 3,38 . The size of the amplifier was $62 \times 31 \mathrm{~mm}$ only. The results of class-B PA are discussed in section-IV.

\section{MEASUREMENT AND OPTIMIZING BIAS POINT}

Assuming that it is desired to reduce the output power of the amplifier (description of the situation in Section I.), it is not enough just to reduce the input signal of amplifier. Lowering of the input power will reduce surplus output power, but at the same time it will shift down on the efficiency curve (Fig. 5a) and the amplifier will have a higher power dissipation.

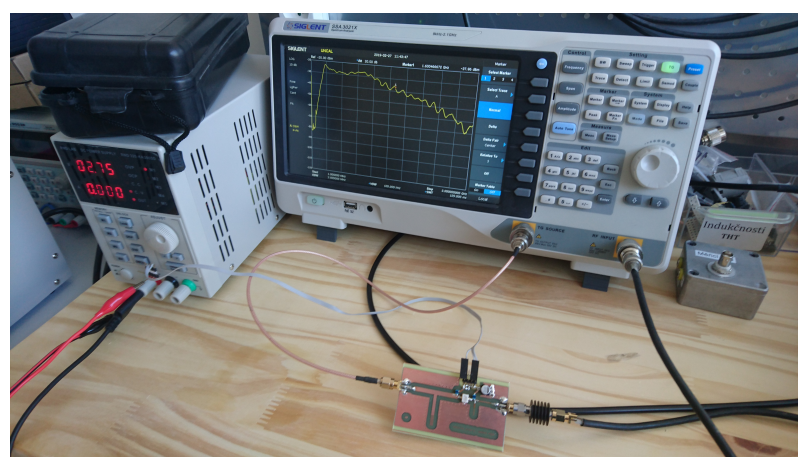

Figure 3: Picture of the developed experimental test bench

Solution of this problem is the optimization of the bias point of amplifier. For RF measurement, a setup was arranged to characterize the amplifier as shown in Fig. 3. RF signal generator is used to apply the input signal and frequency sweep. Attenuator is used to limit RF power to protect spectrum analyzer, while the spectrum is used to analyze the RF output signal. Programmable biasing (-5 V) and drain source $(28 \mathrm{~V})$ are external DC power supplies with OCP (Over Current Protection) were used to bias the amplifier and sequence circuit. In the measurement, RF power sweep was also performed at the centre frequency of 1,6 GHz. The input power was swept from -20 to $0 \mathrm{dBm}$. 
The method is based on a detailed measurement of the entire operating range of the amplifier, where the input power, bias voltage at the gate of transistor, supply voltage and temperature are swept. It is measured during the measurement output power, gate and drain current of transistor. Measurement its an extremely time-consuming process, so it is necessary to do it on an automated workplace. The measurement result resulted in a four dimensional data matrix from which it is possible to calculate the efficiency of the amplifier. On the Fig. 5a. is visualisation of PAE for two different power supplies $28 \mathrm{~V}$ and $19 \mathrm{~V}$.

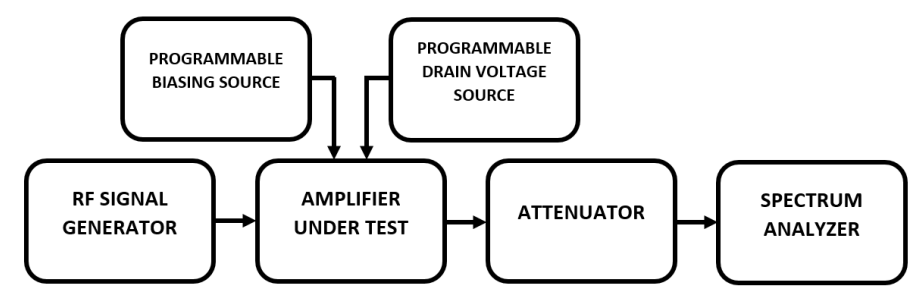

Figure 4: A test-bench setup to characterize the fabricated amplifier

The next step was to create a Matlab script for processing measured data. The algorithm works by going through individual measurements and looking for the best parameters for the required output power.

\section{RESULTS AND DISCUSSION}

After proposed broadband PA design, the simulations were performed prior to the fabrication. The input and output reflection coefficients $\left(\mathrm{S}_{11}\right.$ and $\left.\mathrm{S}_{22}\right)$ are $<-10 \mathrm{~dB}$ at the desired operating frequency $1,6 \mathrm{GHz}$.

The dynamic point adjustment method improves efficiency at low input power by more than $15 \%$ (Fig. 5b). However, with the changing operating conditions of the amplifier (especially the drain power reduction), the output impedance of the transistor changes and the amplifier becomes unadjusted [7]. This problem can be partially compensated by changing the voltage on the gate of transistor, at the cost of a slight reduction of gain. Another important factor that affects the efficiency of the amplifier is the temperature, so it is necessary to calculate with that (especially for aircraft where the temperature is greatly varied) and have it included in the efficiency model of amplifier.

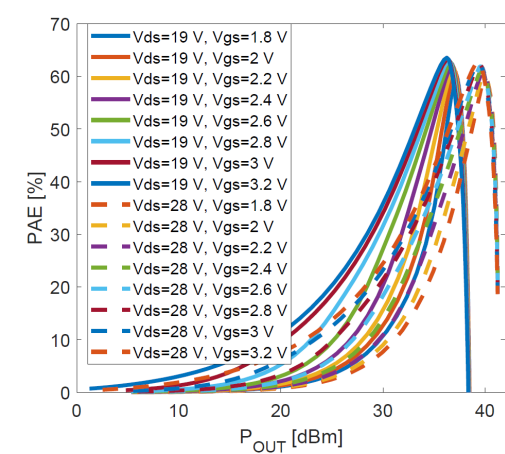

(a)

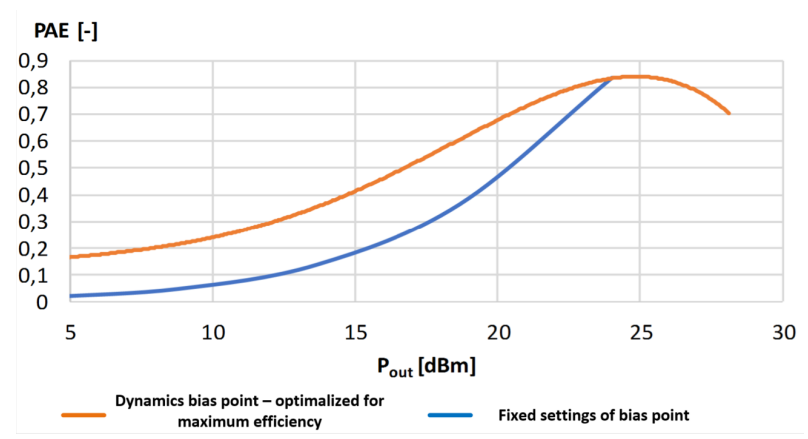

(b)

Figure 5: a) Visualisation of simulated PAE vs Pout at dynamics bias points. b) Measured PAE vs. Pout of fabricated PA at $1.6 \mathrm{GHz}$ with and without dynamic biasing. 
Unlike the envelope tracking, where the envelope of the input signal is extracted by envelope detector and the amplifiers parameters are changed based on its magnitude, the dynamic biasing offers greater efficiency [2] in terms of the energy balance of the broadcasting. The limiting element of both methods is the speed of control of the amplifier parameters. Whilst the Envelope tracking needs to follow the signal envelope very precisely (timing is crucial), and where modulating high supply voltages is challenging, our method take advantage of a priory knowledge about the necessary output power.

\section{CONCLUSION}

In this paper, it was designed and fabricated one-stage c1ass-B amplifier for S-Band (at 1,6 GHz), using the latest GaN on Si HEMT technology. CGH40006P from CREE Corporation was used. The dynamic biasing technique was also described. The proposed method allows to reduce losses by more than $15 \%$ without complex hardware beside fixed settings of the bias point of the class-B amplifier.

\section{ACKNOWLEDGMENT}

This work was supported by the Czech Science Foundation, project no. GA17-18675S and by the BUT project no. FEKTS-17-4426. Research described in this paper was financed by Czech Ministry of Education in frame of National Sustainability Program under grant LO1401. For research, infrastructure of the SIX Center was used.

\section{REFERENCES}

[1] J. Kim, B. Kim and Y. Y. Woo, "Advanced Design of Linear Doherty Amplifier for High Efficiency using Saturation Amplifier", 2007 IEEE MTT-S International Microwave Symposium, pp. 1573-1576, Honolulu, HI, 3-8 June 2007.

[2] P. Medrel, A. Ramadan, J. M. Nebus, P. Bouysse, L. Lapierre and J. F. Villemazet, "High efficiency class $\mathrm{B} \mathrm{GaN}$ power amplifier with dynamic gate biasing for improved linearity," in Electronics Letters, vol. 48, no. 18, pp. 1136-1137, 30 August 2012.

[3] Techplayon.: What is PAPR, Why it matters to Power Amplifier? [online], [cit. 201903-31] Available: http://www.techplayon.com/papr-peak-average-power-ratio-matters-poweramplifier/

[4] Kimball, D.F., et al.: High-efficiency envelope-tracking W-CDMA base-station amplifier using GaN HFETs, IEEE Trans. Microw. Theory Tech., 2006, 54, (11), pp. 3848-3856

[5] A. Kashif, S. Azam, F. Mughal, N. B. Cheema and M. Imran, "Two-stage GaN HEMT based class-C pulsed amplifier for S-band radar applications," 2015 12th International Bhurban Conference on Applied Sciences and Technology (IBCAST), Islamabad, 2015, pp. 560-563.

[6] P. Medrel et al., "Implementation of dual gate and drain dynamic voltage biasing to mitigate load modulation effects of supply modulators in envelope tracking power amplifiers," 2014 IEEE MTT-S International Microwave Symposium (IMS2014), Tampa, FL, 2014, pp. 1-4.

[7] N. Deltimple, L. Leyssenne, E. Kerherve, Y. Deval and D. Belot, "Dynamic biasing techniques for RF power amplifier linearity and efficiency improvement," 2010 IEEE International Conference on Integrated Circuit Design and Technology, Grenoble, 2010, pp. 232-235.

[8] Ceylan, Osman \& Marco, Lazaro \& Pires, Sergio. (2018). Refine biasing networks for high PA low-frequency stability. Microwaves and RF. 57. 52-56. 